\title{
Analysis of Factors Influencing Adoption of Internet of Things: A System Dynamics Approach
}

\author{
Shailja Tripathi' ${ }^{1}$ Laxmi Pandit ${ }^{2}$ \\ ${ }^{1}$ MBA Department of IT and Operations, IFHE University, IBS Hyderabad, Telangana, India \\ ${ }^{2}$ Department of Finance and Operations, IFHE University, IBS Hyderabad, Telangana, India \\ Email: shailja.tripathi@ibsindia.org, laxmi.pandit8@gmail.com
}

How to cite this paper: Tripathi, S. and Pandit, L. (2019) Analysis of Factors Influencing Adoption of Internet of Things: A System Dynamics Approach. Theoretical Economics Letters, 9, 2606-2625. https://doi.org/10.4236/tel.2019.97164

Received: August 1, 2019

Accepted: October 18, 2019

Published: October 21, 2019

Copyright () 2019 by author(s) and Scientific Research Publishing Inc. This work is licensed under the Creative Commons Attribution International License (CC BY 4.0).

http://creativecommons.org/licenses/by/4.0/

\section{(c) () Open Access}

\begin{abstract}
The Internet of things (IoT) has become an interesting topic of research as it assimilates several sensors and objects to communicate directly with one another without human interference. IoT helps businesses and society in many ways such as it improves public safety, transportation, and healthcare by providing better information and faster communications. The adoption of IoT is going to create a persistent impact on society and business. This paper develops a model based on system dynamics and identifies the factors influencing IoT adoption. The dominant factors of IoT adoption are communication, control and automation, efficient business processes, self-configuration, and cost savings. IoT adoption is also affected by negative factors including privacy risks and security risks, interoperability, reliability, poor infrastructure, less skilled IT professionals, etc. Businesses can reap the potential benefits of IoT by solving the issues related to their adoption. Besides, this paper attempts to derive a framework for the successful adoption of IoT in organizations. This study is, therefore, a significant contribution towards understanding the factors that influence the adoption of IoT in businesses.
\end{abstract}

\section{Keywords}

Internet of Things, Reliability, System Dynamics, Self-Configuration, Control and Automation

\section{Introduction}

IoT is an amalgamation of devices, software, sensors, and networks to work jointly to extract significant and usable data or information generated from it [1]. IoT benefits the organization in various ways from improving the efficiency 
of the organization to increase customer satisfaction but the major benefit of IoT that will impact every business is communication, control, and automation, cost savings [2]. IoT helps communicate between the humans and the systems and reduces personnel as well as equipment failure cost [3]. IoT helps in reducing maintenance costs of the plant facilities and increasing production output in a manufacturing firm with the accessibility of real-time data. Transportation and shipping companies are also able to reduce maintenance costs and improve delivery service using IoT [4]. Currently, IoT consists of more desirable sensors, satisfactory computing capability and preferable wireless networks that allow the distribution of IoT application across many sectors of the organization including manufacturing, telecommunication, agriculture, power and energy, health care, supply chain, logistics, data filtering, security and defense industries. The most widely used IoT applications are inter-connected security systems, regulators, cars, electronic machines, electric lights used in houses and commercial places, alarm clocks, speaker systems, vending machines, etc. IoT becomes more powerful when data is generated from various devices and sensors store all in a single location and analyzed statistically and offer new insights and services in real-time. This can be done when IoT integrates with other technologies such as fog computing, edge computing, cloud computing, big data, data mining and machine learning [5]. Fog computing and edge computing help in communicating data in real-time generated by IoT with the help of many communication technologies and protocols. This helps the managers in making real-time decisions in the firms. Cloud computing provides global infrastructure to store this real-time data and data analysis is done using big data technologies such as $\mathrm{Ha}$ doop, NoSQL, and cloud-based Database as a Service (DaaS). Data mining and machine learning or artificial intelligence techniques are used to find out new patterns inside the data. Application developers can utilize this data to create new business applications. In this way, IoT brings business value to a firm by generating a new pattern or insights. IoT also allows firms to enter new markets, enhance the production, streamline their business processes, and satisfies the customer's needs more effectively and efficiently [5].

Various market research firms such as AT \& T emphasized that 30 billion IoT devices will get connected to the Internet by 2020 . Similarly, IDC predicted that several IoT devices for operational usage will be 28.1 billion by 2020 with a CAGR growth of $17.5 \%$ between 2016 and 2020 [6]. Gartner's report predicted that the number of devices connected with IoT will preferably reach by 40 billion and the economic growth of IoT will overreach $\$ 1.9$ billion with 26 billion units by 2020 [7] [8]. A recent study done by Deloitte predicted that, by 2010, IoT devices in India are expected to grow 31 times to 1.9 billion units [9]. Likewise, the NASSCOM report predicted that by 2020 , the Indian market accounted for $5 \%$ of the global market of IoT and expected to grow $\$ 15$ billion [10].

IoT is still in a nascent stage in India and the percentage of organizations with more than fifty thousand connected devices active has been increased two-fold 
in the last year [11]. $81 \%$ of Indian organizations felt that digital transformation occurs due to the evolution of IoT [11]. In India, Industries like manufacturing, logistics, automotive and transportation, and utilities are expected to be the highest adopter of IoT, whereas healthcare, retail, and agriculture industries are predicted to have significant contributions in IoT adoption [12]. The government of India is planned to invest one billion dollars for developing smart cities that influence IoT adoption across the industries. Various big global firms have already adopted IoT like Intel, Microsoft, Samsung, DHL, General Electric, Rolls Royce, Bosch, etc. Some Indian firms have also adopted IoT like HCL Technologies, Infosys, Ramco Systems, and Secure Meters, etc. [12].

Very few studies such as Caro et al. [13]; Hu et al. [14]; Mital et al. [15]; and Mengru Tu [16] have examined IoT adoption in the organization. Prior studies related to IoT adoption highlighted only the factors like perceived usefulness, perceived ease of use, attitude towards adoption, adoption intention, perceived behavioral control, and perceived risks like security/privacy risks. Therefore, the motivation of this study is to identify the other dominant factors that influence IoT adoption by understanding the dynamic behavior of IoT in the organization using a system dynamics model.

This study contributes to the literature by providing a system dynamics model and framework to analyze the factors that influence IoT adoption in the organization. This study fills the gap by introducing other relevant positive and negative factors that influence IoT adoption in the organization. This paper proposes the conceptual model and framework for IoT adoption, therefore future research will be based on formulating hypotheses and data collection. In the future, the proposed model will be tested using advanced econometrical methods like exploratory factor analysis and structural equation modeling.

The paper initiate with the introduction of IoT followed by the purpose and motivation of the study. The literature review of IoT is done in the next section. The conceptual model and framework are discussed in the latter part of the paper. Lastly, the conclusion and implications are discussed in the paper.

\section{Literature Review}

Caro et al. [13] highlighted that IoT plays a vital role in connecting the supply and demand and can act as a retaliatory measure to fill a space between information and fulfillment in the retail industry. IoT adoption generates value by increasing capabilities at a point where supply and demand intersect. Hu et al. [14] articulated and presented IoT in the context of the online retailer's decisions. They throw some light on the positive and negative parts of IoT in the retail industry based on IoT. The retailers could gather various types of information about the customers and analyse the market demand through IoT as well as from other new technologies like big data, Cloud computing. They also highlighted that people are still concerned about personal privacy and (or) health risk caused by the IoT environment. 
Mital et al. [15] explored the intention to adopt IoT in Indian organizations with the help of various theories like Theory of Reasoned Action (TRA), Theory of Planned Behaviour (TPB), and Technology Acceptance Model (TAM). The empirical validation of this model was performed using Structural Equation Modeling. Mengru Tu [16] identified the determinants of IoT adoption intention in logistics and supply chain management using a mixed-method research approach. To understand the impact of IoT on logistics and supply chain management, qualitative analysis was done using the grounded theory methodology. The result showed that perceived benefits, perceived costs, and external pressure are significant factors of IoT adoption intention. The insignificant factors were technology trust.

Olivier et al. [17] developed a security architecture for the Internet of Things (IoT) based on software-defined networking (SDN). SDN was used to achieve network security in an IoT environment. Tarabasz [18] presented the innovative approaches of IoT idea, enumerate association to its opportunities and threats as well as to indicate the inner potential of IoT which is been dominated. With the upcoming opportunities, there are many threats associated with them in the field of information security which requires continuous research and development of new approaches to ensure safety, security, and privacy. Roy et al. [19] validated the underlying factors for the easy adoption of IoT based innovations. They performed the study in four different stages of literature review, survey, interviews with experts and a usability test to develop a model for IoT adoption by the urban poor. The findings showed that IoT based innovation can be in the area of nutrition, healthcare, employment, education, and finances. Kim et al, [20] applied a Value-based model and TAM to analyse the factors that influence the adoption of IoT based smart home service. The findings revealed that perceived value was influenced by both perceived benefit and perceived sacrifice. The study showed that perceived benefit had a stronger positive effect on perceived value than perceived sacrifice.

Tedeschi et al. [21] developed a safe remote monitoring system for machine tools through IoT devices and examines the serious issues focusing on the manufacturing environment. Glória et al. [22] enlightened about the practical implementation of IoT gateway which is dedicated to real-time monitoring and remote control of the swimming pool. Raspberry Pi acts as an aggregate node, Arduino with a set of sensors acts as a sensor node and a web platform to control and maintain the network. A secure system using encrypted communication and an SSL protocol and certificate on a web server-side were developed. Stietencron [23] proposed IoT in the field of marine services to manage the two devices that are automatic identification systems and product embedded information devices to save time and reduce manual efforts. Rahman et al. [24] explained IoT systems as a combination of IoT Device, IoT Service and IoT Application along with the generic life cycle approach model for each of them. They highlighted the development of IoT systems and implementation of generic model into a 
specific IoT system for any specific domain. Boyes et al. [14] reviewed the definition of Industrial IoT. They developed the definition of the Industrial Internet of Things (IIoT) and examined IoT taxonomies. They also developed a framework for IIoT analysis which can be used to compute and describe IIoT devices while reviewing different system architecture. This framework also helped in evaluating security risks and vulnerabilities associated with IIoT. Mir et al. [25] examined the physical and the virtual resource management in IoT systems and highlighted that the basic functioning of IoT includes sensing and data aggradations, processing of the data, storing data and then communication of this data to the virtual environment.

Ray [26] emphasis area-specific architecture of IoT applications and focuses on the challenges and possible research opportunities in the area of defense, military, robotics intelligence services, etc. The author highlighted that IoT may not be adopted by the organization due to its challenges related to flexibility, interoperability, concurrency, scalability, and addressability. Ammar et al. [27] provide a picture of a present state of IoT platforms and identify the trends of different IoT platforms. All the security issues of the IoT framework were considered in terms of authentication, authorization and access control and secure communication.

Chatfield and Reddick [28] have developed a new conceptual framework for IoT enabled smart government. The U.S. federal government's IoT case was examined in four domains: Transportation, Energy, Smart cities and Defence. The finding showed that the strategic usage of IoT is still in the nascent stage in government across the globe. Li et al. [29] review the present research state-of-the-art of 5G IoT, key enabling technologies, and main research trends and challenges in IoT. Pauget and Dammak [30] addressed the organizational implementation of IoT in the case of senior care with three aspects. The first one discusses the patient's independence with the introduction of the Internet of Things; the second one opposes the contradictory image of senior care and the third one confirms endurance among homecare services and institutionalization.

IoT related literature review and a theoretical framework and conceptual model were developed by Nord et al. [31]. The framework highlighted the factors that influence IoT adoption by firms. These studies also highlighted various applications of IoT and found privacy, security, and trust as IoT challenges. Mountrouidou et al. [32] constructed an IoT taxonomy based on generic building blocks for a wider understanding of large pool of IoT devices that are used in home appliances and health care, such as smart plugs, digital assistants, and web cameras, smart meters and robots. They showed that their taxonomy is broad and robust having a simple and effective algorithm that makes it complete, timely, and accurate. Appendix 1 shows the literature review table.

\section{System Dynamics Model}

System dynamics (SD) is a mathematical modeling technique to frame, under- 
stand, and discuss complex issues related to systems [33]. SD is a method to understand the dynamic behaviour of complex systems. Researchers have used SD to design the structure of statistically vigorous systems that put up common dynamics [34]. System dynamics modeling lies in determining the dynamics of a system. The dynamics arise from the interaction of two types of feedback loops positive and negative feedback loops. Positive loops are self-reinforcing that have the capability to amplify whatever happening in the system. Negative loops are self-correcting and have the capability of counteracting and oppose changes in the system. In this study, the causal loop diagram in SD Model is used to identify the positive and negative feedback processes that stimulate IoT adoption. The positive (+) sign at the arrowheads indicates that the effect is positively related to the cause. On the contrary, the negative (-) sign at the arrowheads indicate the effect is negatively related to the cause. The dynamics of the system arise from interactions of networks of these two types of feedback loops.

The system dynamics model was developed and shown in Figure 1. Figure 1 depicts the positive and negative feedback loops in the form of factors that affect IoT adoption in the organization. These factors can be divided into three types: technical, personal and organizational. Personal factor such as privacy risks is negatively influencing IoT adoption. The variables of organization aspects such as cost savings and efficient business processes are positively influencing IoT adoption. The variables of technical aspects such as security, control and automation, communication and self-configuration of IoT devices are also positively influencing IoT adoption. Other technical factors such as reliability, interoperability, poor infrastructure are also negatively influencing IoT adoption. Other organizational factors such as less vendor credibility and less skilled IT professionals also negatively influencing IoT adoption.

The positive factors are communication, control, and automation, cost-saving,

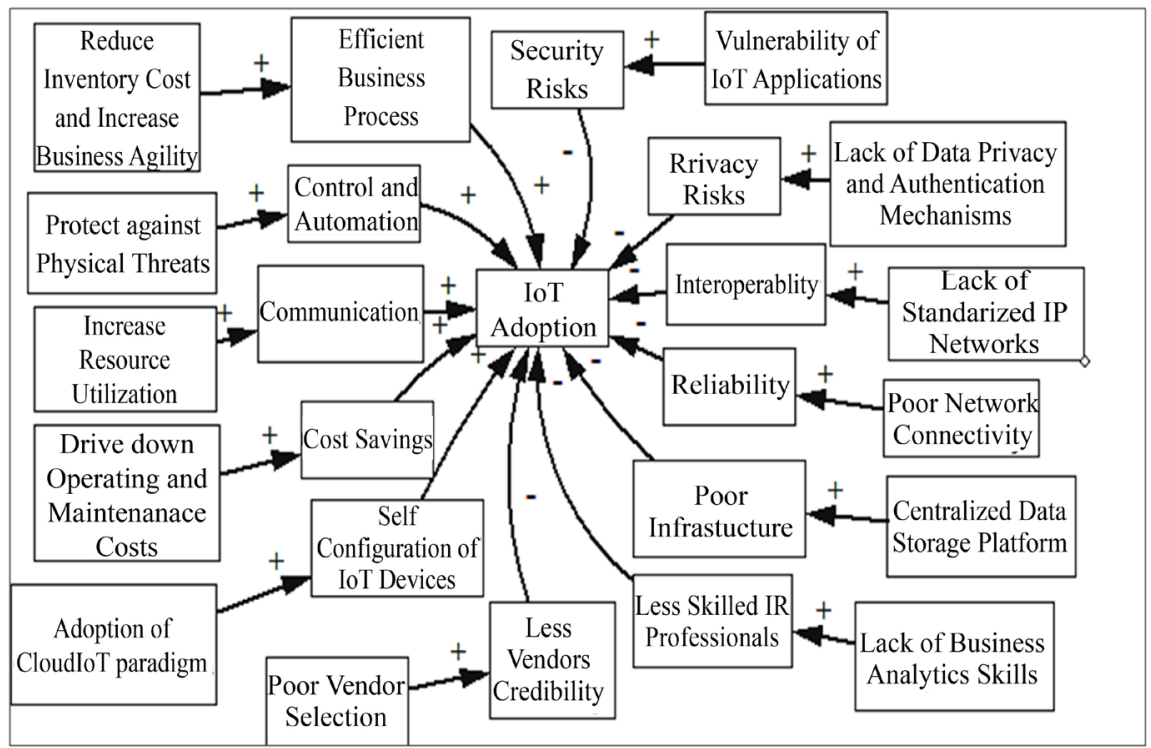

Figure 1. System dynamics model for IoT adoption. 
efficient business processes, and self-configuration of IoT devices. The negative factors are security risks and privacy risks, interoperability, reliability, poor infrastructure, less skilled IT professionals, and less vendor credibility.

\subsection{Analysis of Factors That Positively Influence the Adoption of IoT}

\subsubsection{Communication}

Tracking of assets (such as equipment, machinery, tools, etc.) using sensors and connectivity will be more convenient with IoT, which in turn helps organizations to easily locate issues in the assets and run preventive maintenance to improve asset utilization [2].

Proper resource utilization helps to communicate data and information to people and systems [3]. It also tracks current location and movement like locating items and people within the organisation through sensors like RFID [23]. In this way communication influences IoT adoption in the firm.

\subsubsection{Control and Automation}

IoT also helps the business to provide visibility to send alerts for anomalies and delivered an automated response from the remote device [2]. IoT services assimilated with sensors and video cameras help in ensuring equipment safety and give protection against physical threats in a workplace [2]. In this way control and automation in IoT services synchronize multiple teams to resolve problems on time and encourages IoT adoption.

Caro et al. [13] explained the impact of control and automation in IoT adoption and explained that IoT can be used in three different ways in the demand side of the retail supply chain. First, it can be used as a camera network to analyse customer and employee behaviour and also for inventory management. The second demand-side IoT device used by retailers is smartphones carried by customer and employee to track payment during a store operation. On the demand side, the third option is the usage of the smart cards as loyalty or credit card by the consumers. Finally, on the supply side, IoT is used to track and monitor the inventory on the floor [13].

\subsubsection{Cost Saving}

IoT helps a company to save cost by minimizing equipment failure and allow the business to perform planned maintenance with the help of new sensor information. The return on investment for IoT applications can be easily gained back within a year by enabling capabilities of IoT [13]. IoT also helps to improve resource utilization, business productivity, and increase business process efficiency which in turn cut down operating and maintenance costs [2]. For example, IoT applications such as predictive analytics and real-time diagnostics reduce maintenance costs. In this way, cost-saving promotes IoT adoption in the organization.

\subsubsection{Efficient Business Processes}

Business agility and inventory reduction take place with IoT as it deals with 
real-time operational data [3]. For example, concurrent data collected from logistics network, factory floor, and supply chain will help reduce inventory, time to market and downtime due to inter-connectivity of the maximum number of devices to the internet in IoT platform. Adoption of IoT in manufacturing sector helps to increase business efficiency in its day to day operations and assist them to improve their production quality with high-end safety [3]. Therefore, business process efficiency leads to IoT adoption in the organization.

\subsubsection{Self-Configuration of IoT Devices}

Stergiou et al. [23] suggested that integration of IoT with cloud solutions is required to connect with existing enterprise and operational systems i.e. on public clouds or in private data centers. Maintenance and management of IoT service become easier when IoT service deployed on the cloud platform. IoT platform vendors who are offering a multi-cloud strategy are more responsive to customer and regional requirements. Integration of Cloud with IoT allows IoT devices to integrate with current systems and other IoT devices [35]. This is called the CloudIoT paradigm [35]. Cloud helps IoT to integrate the data collected from its devices with the information exist in current business applications. For example, in a manufacturing company, data collected from sensors on machines can be linked with shipping logs to recognize the influence of machine downtime on delivery times. The cloud plays a major role in the development or improvement of IoT devices because it helps in the automatic configuration of IoT devices securely. Hence, the cloud is an important part of a successful IoT environment. The amalgamation of IoT and Cloud computing provides new prospects of data-sharing and internet-enabled services. This would also lead to a vigorous global network system along with self-configuring capabilities which are based on standard and interoperable communication protocols. According to Distefano et al. [36], IoT devices can securely self-configured and interact with the cloud to customize its behavior. IoT devices can also download the required features from the Cloud. Thus, this integration of cloud platform with IoT would further lead to IoT adoption in the organization.

\subsection{Analysis of Factors That Need to Be Controlled in the Adoption of IoT}

A successful IoT deployment takes place when negative factors are also analysed with the drivers. The main challenges in IoT adoption are security and privacy threats, reliability or poor network connectivity, less skilled IT professionals, infrastructure for storing and analysing data generated by IoT and choosing the right IoT vendor [37].

\subsubsection{Security Risks}

IoT comes with various security issues, such as authorization, verification, access control, system configuration, information storage, and management [16]. According to Voas et al. [38], the security implications of IoT are creating hurdles for its wider adoption. IoT is at high-security risks for several reasons. Firstly, 
IoT systems do not have well-defined standards and are extremely dynamic, unsteady because of mobility. Besides, IoT systems are vastly diverse concerning communication mediums and protocols, platforms, and devices. Furthermore, there can be portability issues as IoT connected devices may not portable to the Internet. Lastly, IoT systems may not be secured [39]. The major IoT security risks involve software attacks that can make use of entire systems, loss of data or information, modify data, refuse service and compromise or spoil devices. According to a survey done by Gartner [4], 32\% of IT leaders highlighted security as a top obstacle to IoT success. Past studies highlight that $70 \%$ of the commonly used IoT devices are at risk because of the lack of software protection, transport encryption, insufficient authorization, and inadequate web interface insecurity [40]. Therefore, there is a need for well-defined policies and a steady legal framework for IoT adoption in the organization [33].

\subsubsection{Privacy Risks}

Kolias et al. [41] focused on privacy threats caused by IoT such as leakage of personally identifiable information (PII) and sensitive user information, and unauthorized execution of functions. Internet of things includes an enormous number of applications that are at present part of people's lives. IoT includes devices in the form of smart objects (SO) that communicate with each other through the Internet. These devices are aggressively delivering the services of IoT applications. This brings privacy concerns to IoT applications because there is a constant increase of involvements of people and devices in these applications.

IoT applications can provide a person's identity and location information to others which may raise privacy concerns. These privacy risks can be simple user irritation in the form of violent advertising such as personalized spam at point-of-sale locations, tracking user routes and constructing user habit profiles and even serious criminal activities [1].

\subsubsection{Interoperability}

Noura et al. [42] highlighted the interoperability as a challenge in IoT adoption in the industry. This challenge can be overcome by connecting IoT devices with standards-based IP networks. The lack of protocols and standards from the governing bodies and the absence of the latest technologies would slow down the adoption of IoT. Furthermore, the integration of IoT and cloud computing would also help in data-sharing and services like self-configuration capabilities based on standard and interoperable communication protocols.

\subsubsection{Infrastructure}

With the incremental growth in real-time data, companies need to opt for a sustainable solution for data storage. The real-time data are stored centrally which is unsustainable. Hence, companies should switch to a distributed cloud platform from centralized data storage [37]. Also, companies should ensure that they have the right infrastructure that manages IoT connected devices, sensors, and data generating in real-time. 


\subsubsection{Network Connectivity/Reliability}

The successful IoT adoption can be ensured by powerful and reliable network connectivity [37]. Instead of establishing proprietary networks, IoT devices should be connected with standards-based IP networks. IoT service can be delivered with performance with an IP-based network without the issues of reliability and interoperability.

\subsubsection{Less Skilled IT Professionals}

IoT is a very extensive area and generates a huge volume of data collected from several sources using devices and sensors. Therefore companies require IT professionals with big data analytical skills to make quick and reliable business decisions. An IT professional should have sufficient analytical skills in business intelligence areas such as data center management, sensor data analysis, predictive analytics, with programming knowledge of NoSQL distributed databases and Hadoop file system that are required to manage huge and fast-growing data sets. Therefore, there is a need for a team of IT professionals with business analytical skills for successful IoT adoption in the organization [37].

\subsubsection{Vendors Credibility}

Today, organizations outsource IoT solutions to the third-party vendor. The selection of IoT vendor is one of the most crucial decisions which businesses take. IoT platforms that offer the right infrastructure, high scalability, optimized results, and disaster recovery, without compromising on precision and efficiency are required [37]. Therefore, the challenge is to find out the appropriate IoT vendor that provides the best IoT solution.

Table 1 shows the factors that need to control along with the remedies in the successful adoption of IoT in the organization.

\section{The Framework of Adoption of IoT in an Organization}

The framework was developed and shown in Figure 2. The framework shows

Table 1. Factors to be controlled along with their measures to control in IoT adoption.

\begin{tabular}{|c|c|}
\hline Factors to be controlled & Measures to control \\
\hline Privacy Risks & $\begin{array}{l}\text { Identity and authentication structures need to be updated time } \\
\text { to time. }\end{array}$ \\
\hline Security Risks & $\begin{array}{l}\text { Security procedures such as hardware encryption, physical } \\
\text { building security and network security need to be implemented. }\end{array}$ \\
\hline Infrastructure & $\begin{array}{l}\text { Sustainable solutions for data storage need to be implemented } \\
\text { like Cloud Computing. }\end{array}$ \\
\hline Reliability/Network Connectivity & Establish powerful and reliable network connectivity. \\
\hline Interoperability & Connect IoT devices with standards-based IP networks. \\
\hline Less skilled IT professionals & $\begin{array}{l}\text { A team of IT professional with knowledge of business analytics } \\
\text { tools. }\end{array}$ \\
\hline Choose the right vendor & $\begin{array}{l}\text { Find the best IoT vendor that provide right IoT infrastructure } \\
\text { with scalability, disaster recovery, precision and efficiency. }\end{array}$ \\
\hline
\end{tabular}




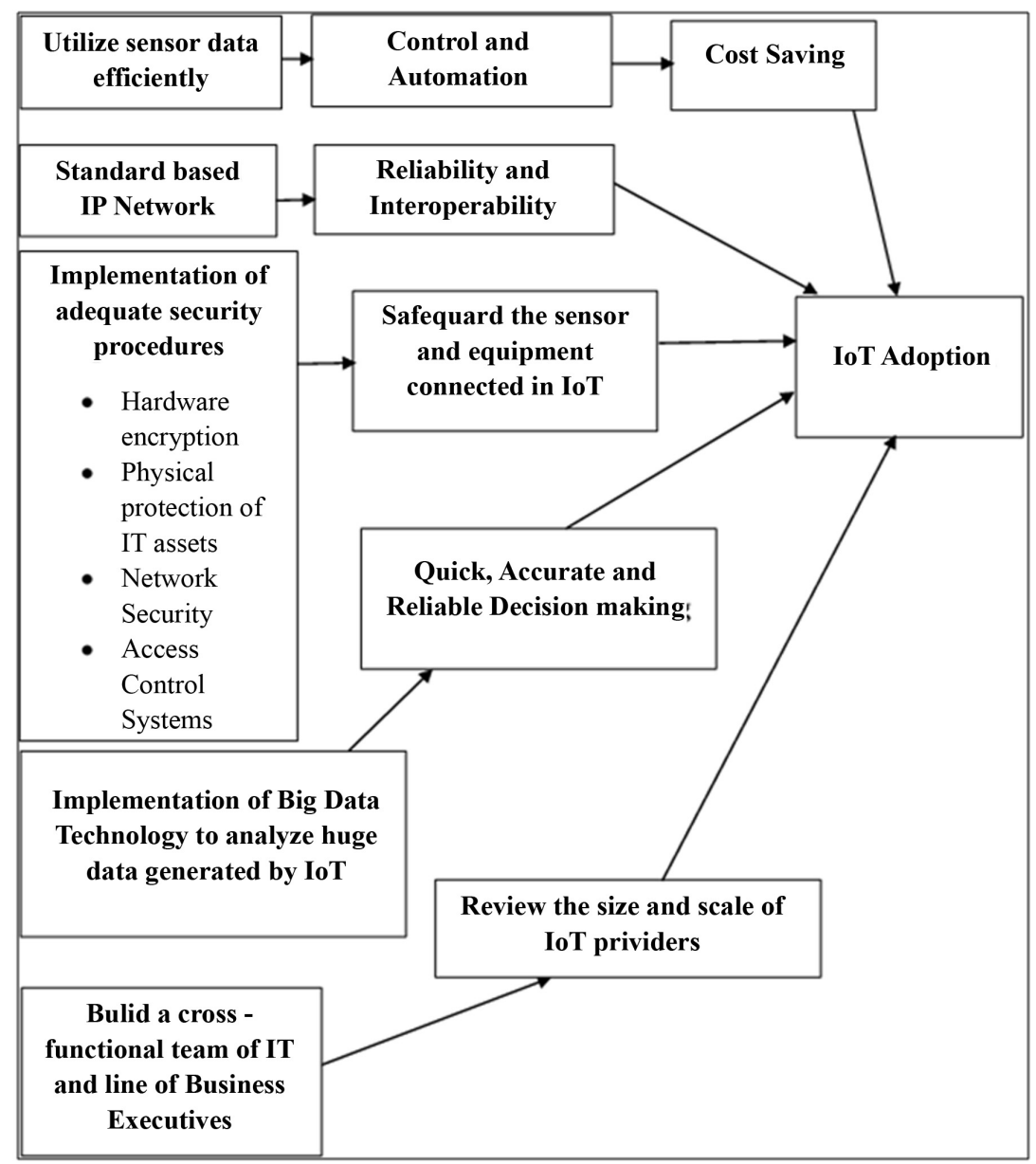

Figure 2. A framework of adoption of IoT in an organization.

the different factors and sub-factors that influence IoT adoption. In case of cost saving, with new sensor information, IoT can help a company, save money by minimizing equipment failure and allowing the business to perform planned maintenance. IoT will improve tracking of assets (equipment, machinery, tools, etc.) using sensors and connectivity, which helps organizations benefit from real-time insights. With the help of IoT, organizations could more easily find problems in the assets and execute preventive maintenance to get better asset utilization. IoT helps in reducing operating costs by providing real-time operational insights to the organization. For example, in a manufacturing firm, IoT collects data from the logistics network, factory floor, and supply chain. This will help the firm to reduce inventory, time to market and downtime due to maintenance. The improved asset utilization, productivity, and process efficiencies can save operating and maintenance costs.

Implementation of security procedures such as hardware encryption, physical building security, and network security are needed to safeguard the sensor and equipment connected in IoT. Identity and authentication structures will also need to be updated to minimize the privacy risks associated with IoT in the organization. According to Nord et al. [31], security decisions maker is unknown 
in IoT area because there are many partners involved in IoT including designing a device, supplies software component, operation of a network where devices are embedded, deployment of the device. There is a need for comprehensive, international norms Successful IoT adoption only occurs when security and privacy risks are minimized.

Successful IoT adoption takes place when IoT devices are connected with standards-based IP networks instead of using proprietary networks for communication. Interoperability and reliability are required to support global IoT networks and connections with partner ecosystems.

Organizations must build a data collection and analytics strategy that supports the new and large amount of information in a scalable and cost-effective manner, generated by IoT. Cloud computing provides an IoT infrastructure for data storage. Big data technology, such as Hadoop and NoSQL, can give companies the ability to rapidly collect, store and analyze large volumes of disparate IoT data. Cloud computing and big data technologies help the managers to make quick, accurate and reliable decisions in the business. This leads to the successful adoption of IoT.

IoT is a complex landscape with numerous categories and many vendors within each category. The four main categories of an IoT solution are sensors or radios that fit inside the device, an M2M device management platform, a solution delivery platform and applications that enable IoT devices to act on data, generated by sensors. This landscape requires many vendors for IoT because no single vendor offers a complete solution without building partnerships. This requires a cross-functional team of IT professionals and business executives to evaluate the financial position of the vendors, their industry knowledge, partnerships and their range of offerings, which in turn helps in IoT adoption in an organization.

\section{Conclusions and Implications}

This study portrays the positive and negative factors of IoT adoption with the help of the system dynamics model. The model gives a clear picture of technical, personal and organizational factors of IoT adoption. Personal factor consists of privacy risks that negatively influence IoT adoption. Cost savings and efficient business processes are the organizational factors that positively influence IoT adoption. Technical factors such as control and automation, communication and self-configuration of IoT devices are positively influencing IoT adoption. Other technical factors such as security, reliability, interoperability, poor infrastructure are negatively influencing IoT adoption. Less skilled IT professionals and vendor credibility are other organizational factors that have a negative impact on IoT adoption.

Although IoT offers immense potential value, organizations must find solutions for some significant challenges related to IoT adoption such as data and information management issues, lack of interoperable technologies, security and 
privacy concerns, and the skills to manage a growing and complex landscape of IoT. Conversely, a professional IoT vendor can overcome these challenges and increase return on investment of the companies. This study is, therefore, a significant contribution towards understanding the factors that influence the adoption of innovations like IoT in businesses. To implement IoT successfully and to analyse the hindrances, companies should make use of modern technologies like fog/edge computing, cloud computing, big data technology, data mining and machine learning with the help of highly skilled IT professionals. Currently, IoT is used to connect several devices in a network for commercial and domestic purposes. Therefore, there is a major influence of IoT on society and business.

A review of the literature showed that IoT is still an emerging area of research. Based on the literature review of available research papers and white papers, a system dynamics model of IoT adoption and conceptual framework are proposed. Analysis of the positive and negative factors that influence IoT adoption in the organization was done with the help of the system dynamics model. This study contributes to the literature by exploring additional factors of IoT adoption and also by proposing the framework of successful IoT adoption in the organization. This study fills the gap by introducing other relevant positive and negative factors that influence IoT adoption in the organization. Future research will be the focus on empirically testing of the conceptual model by collecting data using a questionnaire. Advanced statistical analytical tools like SPSS Statistics Version 26 will be used to validate the model.

\section{Conflicts of Interest}

The authors declare no conflicts of interest regarding the publication of this paper.

\section{References}

[1] Samani, A., Ghenniwa, H.H. and Wahaishi, A. (2015) Privacy in Internet of Things: A Model and Protection Framework. Procedia Computer Science, 52, 606-613. https://doi.org/10.1016/j.procs.2015.05.046

[2] https://www.cisco.com/c/dam/en_us/solutions/trends/iot/introduction_to_IoT_nov ember.pdf

[3] https://vmokshagroup.com/blog/6-ways-businesses-can-take-advantage-of-iot

[4] https://www.fujitsu.com/global/imagesgig5/fujitsu_iot_infograhpic_final_250918.p df

[5] https://www.forbes.com/sites/mikekavis/2016/02/25/iot-is-the-killer-app-for-big-da ta/\#1ea9356e11fa

[6] https://softwarestrategiesblog.com/tag/internet-of-things-market-estimates/

[7] https://tech.economictimes.indiatimes.com/news/corporate/25-billion-connected-t hings-will-be-in-use-by-2021-gartner/66563141

[8] https://www.gartner.com/smarterwithgartner/the-iot-effect-opportunities-and-chall enges-2

[9] https://economictimes.indiatimes.com/tech/internet/iot-techs-big-billion-days-arec 
oming/articleshow/69886336.cms?from $=\mathrm{mdr}$

[10] https://www.mendix.com/examples-of-iot-applications

[11] Gurnaney (2018) Future Picture: Why India Will Need Internet of Things to Bring in Major Transformation.

https://telecom.economictimes.indiatimes.com/news/future-picture-why-india-willneed-internet-of-things-to-bring-in-major-transformation/61729747

[12] https://www.livemint.com/Technology/AvA3BgHQO0QnLoEPYUu9ZK/Why-Inter net-of-Things-can-become-the-game-changer-that-Indi.html

[13] Caro, F. and Sadr, R. (2019) The Internet of Things (IoT) in Retail: Bridging Supply and Demand. Business Horizons, 62, 47-54. https://doi.org/10.1016/j.bushor.2018.08.002

[14] Hu, S., Hu, B. and Cao, Y. (2018) The Wider, the Better? The Interaction between the IoT Diffusion and Online Retailers' Decisions. Physica A: Statistical Mechanics and Its Applications, 509, 196-209. https://doi.org/10.1016/j.physa.2018.06.008

[15] Mital, M., Chang, V., Choudhary, P., Papa, A. and Pani, A.K. (2018) Adoption of Internet of Things in India: A Test of Competing Models Using a Structured Equation Modeling Approach. Technological Forecasting and Social Change, 136, 339-346. https://doi.org/10.1016/j.techfore.2017.03.001

[16] Tu, M. (2018) An Exploratory Study of Internet of Things (IoT) Adoption Intention in Logistics and Supply Chain Management: A Mixed Research Approach. The International Journal of Logistics Management, 29, 131-151. https://doi.org/10.1108/IJLM-11-2016-0274

[17] Olivier, F., Carlos, G. and Florent, N. (2015) New Security Architecture for IoT Network. Procedia Computer Science, 52, 1028-1033.

https://doi.org/10.1016/j.procs.2015.05.099

[18] Tarabasz, A. (2016) The Internet of Things-Digital Revolution in Offline Market. Opportunity or Threat? Handel Wewnętrzny, 4, 325-337.

[19] Roy, A., Zalzala, A.M.S. and Kumar, A. (2016) Disruption of Things: A Model to Facilitate Adoption of IoT-Based Innovations by the Urban Poor. Procedia Engineering, 159, 199-209. https://doi.org/10.1016/j.proeng.2016.08.159

[20] Kim, T.H., Ramos, C. and Mohammed, S. (2017) Smart City and IoT. Future Generation Computer Systems, 76, 159-162. https://doi.org/10.1016/j.future.2017.03.034

[21] Tedeschi, S., Mehnen, J., Tapoglou, N. and Roy, R. (2017) Secure IoT Devices for the Maintenance of Machine Tools. Procedia CIRP, 59, 150-155. https://doi.org/10.1016/j.procir.2016.10.002

[22] Glória, A., Cercas, F. and Souto, N. (2017) Design and Implementation of an IoT Gateway to Create Smart Environments. Procedia Computer Science, 109, 568-575. https://doi.org/10.1016/j.procs.2017.05.343

[23] Stergiou, C., Psannis, K.E., Kim, B.G. and Gupta, B. (2018) Secure Integration of IoT and Cloud Computing. Future Generation Computer Systems, 78, 964-975. https://doi.org/10.1016/j.future.2016.11.031

[24] Rahman, L.F., Ozcelebi, T. and Lukkien, J. (2018) Understanding IoT Systems: A Life Cycle Approach. Procedia Computer Science, 130, 1057-1062. https://doi.org/10.1016/j.procs.2018.04.148

[25] Boyes, H., et al. (2018) The Industrial Internet of Things (IIoT): An Analysis Framework. Computers in Industry, 101, 1-12. https://doi.org/10.1016/j.compind.2018.04.015 
[26] Mir, R.N. (2018) Resource Management in Pervasive Internet of Things: A Survey. Journal of King Saud University_Computer and Information Sciences.

[27] Ray, P.P. (2018) A Survey on Internet of Things Architectures. Journal of King Saud University-Computer and Information Sciences, 30, 291-319. https://doi.org/10.1016/j.jksuci.2016.10.003

[28] Ammar, M., Russello, G. and Crispo, B. (2018) Internet of Things: A Survey on the Security of IoT Frameworks. Journal of Information Security and Applications, 38, 8-27. https://doi.org/10.1016/j.jisa.2017.11.002

[29] Chatfield, A.T. and Reddick, C.G. (2018) A Framework for Internet of Things-Enabled Smart Government: A Case of IoT Cybersecurity Policies and Use Cases in US Federal Government. Government Information Quarterly, 36, 346-357. https://doi.org/10.1016/j.giq.2018.09.007

[30] Pauget, B. and Dammak, A. (2019) The Implementation of the Internet of Things: What Impact on Organizations? Technological Forecasting and Social Change, 140, 140-146. https://doi.org/10.1016/j.techfore.2018.03.012

[31] Nord, J.H., Koohang, A. and Paliszkiewicz, J. (2019) The Internet of Things: Review and Theoretical Framework. Expert Systems with Applications, 133, 97-108. https://doi.org/10.1016/j.eswa.2019.05.014

[32] Mountrouidou, X., Billings, B. and Mejia-Ricart, L. (2019) Not Just Another Internet of Things Taxonomy: A Method for Validation of Taxonomies. Internet of Things, 6, Article ID: 100049. https://doi.org/10.1016/j.iot.2019.03.003

[33] Hsu, A.P., Lee, W.T., Trappey, A.J., Trappey, C.V. and Chang, A.C. (2015) Using System Dynamics Analysis for Performance Evaluation of IoT Enabled One-Stop Logistic Services. IEEE International Conference on Systems, Man, and Cybernetics, Hong Kong, 9-12 October 2015, 1291-1296. https://doi.org/10.1109/SMC.2015.230

[34] Qu, T., Thürer, M., Wang, J., Wang, Z., Fu, H., Li, C. and Huang, G.Q. (2017) System Dynamics Analysis for an Internet-of-Things-Enabled Production Logistics System. International Journal of Production Research, 55, 2622-2649. https://doi.org/10.1080/00207543.2016.1173738

[35] Botta, A., De Donato, W., Persico, V. and Pescapé, A. (2014) On the Integration of Cloud Computing and Internet of Things. International Conference on Future Internet of Things and Cloud, Vienna, 22-24 August 2016, 23-30. https://doi.org/10.1109/FiCloud.2014.14

[36] Distefano, S., Merlino, G. and Puliafito, A. (2015) A Utility Paradigm for IoT: The Sensing Cloud. Pervasive and Mobile Computing, 20, 127-144. https://doi.org/10.1016/j.pmcj.2014.09.006

[37] https://www.allerin.com/blog/due-to-these-5-barriers-companies-hesitate-to-adopt$\underline{\text { iot }}$

[38] Voas, J. and Laplante, P.A. (2017) The IoT Blame Game. Computer, 50, 69-73. https://doi.org/10.1109/MC.2017.169

[39] Bertino, E. (2016) Data Privacy for IoT Systems: Concepts, Approaches, and Research Directions. IEEE International Conference on Big Data, Washington DC, 5-8 December 2016, 3645-3647. https://doi.org/10.1109/BigData.2016.7841030

[40] Lee, I. and Lee, K. (2015) The Internet of Things (IoT): Applications, Investments, and Challenges for Enterprises. Business Horizons, 58, 431-440. https://doi.org/10.1016/j.bushor.2015.03.008

[41] Kolias, C., Stavrou, A., Voas, J., Bojanova, I. and Kuhn, R. (2016) Learning Internet-of-Things Security "Hands-On". IEEE Security \& Privacy, 14, 37-46. 
https://doi.org/10.1109/MSP.2016.4

[42] Noura, M., Atiquzzaman, M. and Gaedke, M. (2018) Interoperability in Internet of Things: Taxonomies and Open Challenges. Mobile Networks and Applications, 24, 796-809. 


\section{Appendix 1. Literature Review Table}

\begin{tabular}{|c|c|c|c|c|}
\hline Title/Journal & Author & Year & Model & Objective \\
\hline $\begin{array}{l}\text { The implementation of the } \\
\text { Internet of Things: What } \\
\text { impact on organizations? }\end{array}$ & $\begin{array}{l}\text { Bertrand Pauget, Ahmed } \\
\text { Dammak }\end{array}$ & 2019 & Literature Review & $\begin{array}{l}\text { This study proposed a model to understand the } \\
\text { impact of implementation of IoT technology in the } \\
\text { organization like senior care sector. }\end{array}$ \\
\hline $\begin{array}{l}\text { Internet of Things (IoT) in } \\
\text { retail: Bridging supply and } \\
\text { demand }\end{array}$ & Felipe Caro, Ramin Sadr & 2019 & Literature Review & $\begin{array}{l}\text { This study classified IoT initiatives on an opportunity } \\
\text { map and presented a strategic framework that } \\
\text { distinguishes initiatives by the value they create } \\
\text { and by their major area of impact on retail firms. }\end{array}$ \\
\hline $\begin{array}{l}\text { 5G Internet of Things: A } \\
\text { survey }\end{array}$ & $\begin{array}{l}\text { Shancang Li, Li Da Xu, } \\
\text { ShanshanZhaof }\end{array}$ & 2018 & Literature Review & $\begin{array}{l}\text { This paper reviews the current research } \\
\text { state-of-the-art of } 5 \mathrm{G} \text { IoT, key enabling } \\
\text { technologies, and main research trends and } \\
\text { challenges in } 5 \mathrm{G} \text { IoT. }\end{array}$ \\
\hline $\begin{array}{l}\text { A framework for Internet of } \\
\text { Things-enabled smart } \\
\text { government: A case of IoT } \\
\text { cybersecurity policies and use } \\
\text { cases in U.S. federal } \\
\text { government }\end{array}$ & $\begin{array}{l}\text { Akemi Takeoka Chatfield, } \\
\text { Christopher G. Reddick }\end{array}$ & 2018 & Literature Review & $\begin{array}{l}\text { This paper developed a framework for IoT-enabled } \\
\text { smart government performance. This study applied } \\
\text { the framework to conduct case study analyses of } \\
\text { digital technology policy, IoT cybersecurity policy, } \\
\text { and IoT use in major application domains at the } \\
\text { U.S. federal government level. }\end{array}$ \\
\hline $\begin{array}{l}\text { A survey on Internet of } \\
\text { Things architectures }\end{array}$ & P.P. Ray & 2018 & $\begin{array}{l}\text { Organizational } \\
\text { theory }\end{array}$ & $\begin{array}{l}\text { In this article, firstly the background and definition } \\
\text { of IoT are given. Secondly, thorough discussions } \\
\text { on fundamentals behind IoT architectures are } \\
\text { elaborated. }\end{array}$ \\
\hline $\begin{array}{l}\text { The industrial internet of } \\
\text { things (IIoT): An analysis } \\
\text { framework }\end{array}$ & $\begin{array}{l}\text { Hugh Boyes, BilHallaq, Joe } \\
\text { Cunningham, Tim Watson }\end{array}$ & 2018 & Literature Review & $\begin{array}{l}\text { This paper reviews what is meant by Industrial IoT } \\
\text { (IIoT) and relationships to concepts such as } \\
\text { cyber-physical systems and Industry 4.0. The paper } \\
\text { develops a definition of IIoT and analyses related } \\
\text { partial IoT taxonomies. }\end{array}$ \\
\hline $\begin{array}{l}\text { Understanding IoT Systems: } \\
\text { A Life Cycle Approach }\end{array}$ & $\begin{array}{l}\text { Leila Fatmasari Rahmana, } \\
\text { Tanir Ozcelebia, Johan } \\
\text { Lukkien }\end{array}$ & 2018 & Literature Review & $\begin{array}{l}\text { This study defined a generic life cycle model for } \\
\text { IoT, which is specified by observations on life } \\
\text { cycles of existing IoT solutions and generalization. }\end{array}$ \\
\hline $\begin{array}{l}\text { Success of IoT in Smart Cities } \\
\text { of India: An empirical analysis }\end{array}$ & $\begin{array}{l}\text { Sheshadri Chatterjee, Arpan } \\
\text { Kumar Kar, M.P. Gupta }\end{array}$ & 2018 & $\begin{array}{c}\text { Updated } \\
\text { information } \\
\text { system success } \\
\text { model }\end{array}$ & $\begin{array}{l}\text { The study identifies key factors affecting successful } \\
\text { implementation of information system in Smart } \\
\text { Cities of India (SCI). This study also highlights } \\
\text { successful implementation of IoT policy in Smart } \\
\text { Cities of India coupled with Artificial Intelligence. }\end{array}$ \\
\hline $\begin{array}{l}\text { Internet of Things: A survey } \\
\text { on the security of IoT } \\
\text { frameworks }\end{array}$ & $\begin{array}{l}\text { Mahmoud Ammar, Giovanni } \\
\text { Russello, Bruno Crispo }\end{array}$ & 2018 & Literature Review & $\begin{array}{l}\text { This study gave a high overview of the current state } \\
\text { of the art IoT platforms. This study also provided a } \\
\text { high level comparison between the different } \\
\text { security architectures of the various frameworks. }\end{array}$ \\
\hline $\begin{array}{c}\text { A Survey of How to Use } \\
\text { Blockchain to Secure Internet } \\
\text { of Things and the Stalker } \\
\text { Attack }\end{array}$ & $\begin{array}{l}\text { Emanuel Ferreira Jesus, } \\
\text { Vanessa R. L. Chicarino, } \\
\text { Célio V. N. de Albuquerque, } \\
\text { and Antônio A. de A. Rocha }\end{array}$ & 2018 & Literature Review & $\begin{array}{l}\text { The paper goal is to provide the concepts about the } \\
\text { structure and operation of Blockchain and, mainly, } \\
\text { analyse how the use of this technology can be used } \\
\text { to provide security and privacy in IoT. }\end{array}$ \\
\hline $\begin{array}{l}\text { Resource management in } \\
\text { pervasive Internet of Things: } \\
\text { A survey }\end{array}$ & $\begin{array}{c}\text { Saniya Zahoor, RoohieNaaz } \\
\text { Mir }\end{array}$ & 2018 & Literature Review & $\begin{array}{l}\text { This paper presents a survey of physical and virtual } \\
\text { resource management in IoT systems. The main } \\
\text { focus of the paper is on resource management in } \\
\text { pervasive IoT environment with limited resources. }\end{array}$ \\
\hline $\begin{array}{l}\text { Internet of Things: A } \\
\text { systematic review of the } \\
\text { business literature from the } \\
\text { user and organisational } \\
\text { perspectives }\end{array}$ & $\begin{array}{l}\text { Yang Lu, SavvasPapagiannidis, } \\
\text { EleftheriosAlamanos }\end{array}$ & 2018 & Literature Review & $\begin{array}{l}\text { The study followed a systematic approach to } \\
\text { reviewing the relevant literature following a } \\
\text { number of steps, such as planning the review, } \\
\text { selecting and reviewing the papers, synthesising the } \\
\text { results, and reporting the findings. }\end{array}$ \\
\hline
\end{tabular}




\section{Continued}

Understanding business model in the Internet of Things industry

Intertwining the internet of things and consumers' behaviour science: Future promises for businesses

An experimental study of a Byungseok Kang Hyunseung reliable IoT gateway

Secure IoT Devices for the Maintenance of Machine Tools

Stefano Tedeschia, Jörn Mehnena, Nikolaos Tapogloua, Rajkumar Roya

Adel Alkhalil, Rabie A. Ramadan
IoT Data Provenance Implementation Challenges

Design and implementation of an IoT gateway to create smart environments

Andr'eGl'oria, Francisco Cercas, NunoSouto

The Internet of Things: Are Ted Saarikko, Ulrika H. you ready for what's coming? Westergren, Tomas Blomquist

Christian Røstad,

Things for the Management of

Through-life Engineering

Services on Marine Auxiliaries

Bjørnar Henriksen,

Klaus-Dieter Thoben

\section{Adoption of Internet of}

Things in India: A test of Monika Mital, Victor Chang, competing models using a Praveen Choudhary, Armando structured equation modeling

Papa, Ashis K. Pani approach
Literature Review

\section{w} support for industry standards and interoperability of devices, ability to solve the challenges of the information link, secure clouds for traditional systems.

The paper presents a proposal of a practical implementation for an IoT gateway dedicated to real-time monitoring and remote control of a

Using an explorative sequential approach through the multiple-case study method, we apply the business model of three companies operating in IoT industry, namely Intel, Solair, and Apio.

The objective of this study is to offer an explor-

Empirical paper ative, quantitative study on the IoTs to enhance the (motivation the- existing knowledge and support business in the process of engaging more users and creating new personalised products.

This paper introduced an experimental study of a reliable and self-configurable IoT gateway that is developed in a laboratory testbed by using the IoTivity framework.

This paper aims to establish a starting point towards addressing security and privacy challenges of IoT by suggesting the implementation of data provenance in IoT.

This paper introduced a global strategy for secure Design for IoT which includes: safe solutions for environments with rich information, guarantee that the devices are functioning as intended by the manufacturer and are not damaged, life cycle swimming pool. Based on a Raspberry Pi, the

2017 Conceptual paper gateway allows bidirectional communication and data exchange between the user and the sensor network implemented on the environment using an Arduino.

This study reviewed the complexity of the IoT, the complexities of an increasingly interconnected

Literature Review environment, and the increasing need to develop partnerships in order to create IoT based innovative solutions.

This paper presents a solution approach and its prototypical implementation utilising the Internet Literature Review of Things (IoT) to aid the marine auxiliaries' producers in the process of managing the product usage phase and its services.

This study explored the adoption of Internet of

Empirical Things from a multiple theory perspective, namely,

2017 Paper-Structured The Theory of Reasoned Action (TRA), The Equation Modeling Theory of Planned Behavior (TPB) and The Technology Acceptance Model (TAM). 


\section{Continued}

The Framework of Business Model in the Context of Industrial Internet of Things

The Internet of Things and new business opportunities

The Internet of

Things-Chance and challenge in industrial business relationships

Christine Falkenrecka, Ralf Wagner

The influence of the Industrial Internet of Things on business models of established manufacturing companies-A Daniel Kiel, Christian Arnold, business level perspective

Challenges and Solutions for Applications and

Technologies in the Internet of Things

Disruption of Things: A Model to Facilitate Adoption of IoT-based Innovations by the Urban Poor

The Internet of

Things-Digital Revolution in Offline Market.

Anna Tarabasz

SaadAlbishi

Ben Soh

AzmatUllah

Fahad Algarni

Abhimanyu Roy, Ali M.S.Zalzala, Alok Kumar

Opportunity or Threat?

Prototyping Business Models Jaehyeon Jua, Mi-Seon Kima, for IoT Service Jae-Hyeon Ahn

Management and Internet of Things

Mayra Samaniego, Ralph Deters

New Security Architecture for IoT Network

Olivier, Gonzalez Carlos, Nolot Florent

An Integrated Approach to

Common Problems in the Internet of Things
Sergey Efremov, Nikolay Pilipenko, Leonid Voskov
2017 2016
Conceptual paper

The purpose of this article is an attempt to develop the concept of a business model dedicated to companies implementing technologies of the Industrial Internet of Things.

This study elaborated the business opportunities provided by IoT by covering various elements within the technological, physical, and socioeconomic environments that comprise IoT.

This study developed and discussed a conceptual frame of organizational networks, trust, and

Multi-method technology acceptance in the context of the IoT. research approach The empirical data provide evidence of how IoT transactions affect trust in buyer-manufacturer relationships.

Qualitative empirical approach This study aims at analysing the influence of the (Semi-structured Industrial Internet of Things (IIoT) on established expert interviews) business models of manufacturing companies.

The aim of this paper is to study the literature

Literature Review review of electronics applications in the context of the Internet of Things' challenges and solutions for emerging sensors and electronics applications.

Literature Review, This study examines the adoption of the Internet of Interviews and Things (IoT) based innovations by urban poor Usability test communities.

The main aim of this article is to present the innovative approach of the IoT idea, enumerate

Literature Review juxtaposition of its opportunities and threats as well as to indicate its inner potential still being dormant.

The aim of this research is to develop a generic business model framework for IoT business Case study through literature analysis and interviews. To test the proposed business model framework, case studies of current IoT companies had done.

This work addressed the management of resources in the Internet of Things. This is achieved by

Conceptual paper proposing a virtual-resource edge layer, which enables access and configuration to constrained physical resources.

This study explained the notion of security Literature Review architecture for Internet of Things (IoT) based on software-defined networking (SDN).

The study investigated the topic and point out 2015 Literature Review several problems that need to be efficiently solved for the Internet of Things to work on large scale numbers. 


\section{Continued}

Business models for the Internet of Things

A distributed and flexible architecture for Internet of Things

Business Models for the Internet of Things Environment

R.M. Dijkmana, B. Sprenkels,

Ghofrane Fersi T. Peeters, A. Janssen

2015 2015

Jozef Glova, Tomáš Sabol, ViliamVajda
This paper presented a framework for developing business models for IoT applications. The framework is created based on a literature survey Literature Review into existing business model frameworks and subsequently adapting these frameworks based on interviews in 11 companies that develop IoT applications.

This article presented a new approach for use in the Internet of Things. It includes a complete Conceptual paper architecture that integrates things identifiers assignment, routing, churn and mobility management.

This paper provides an overview of business 2014 Conceptual Paper models for Internet of Things, Services and People applications. 